\title{
Nitric oxide, interorganelle communication, and energy flow: a novel route to slow aging
}

\author{
Alessandra Valerio ${ }^{1}$ and Enzo Nisoli $^{2 *}$ \\ Department of Molecular and Translational Medicine, University of Brescia, Brescia, Italy \\ ${ }^{2}$ Department of Medical Biotechnology and Translational Medicine, Center for Study and Research on Obesity, University of Milan, Milan, Italy
}

\section{Edited by:}

Paul Huang, Massachusetts General

Hospital, USA

Reviewed by:

Bradford G. Hill, University of

Louisville, USA

Lufang Zhou, The University of

Alabama at Birmingham, USA

*Correspondence:

Enzo Nisoli, Department of Medical Biotechnology and Translational

Medicine, University of Milan, via

Vanvitelli 32, 20129 Milan, Italy

e-mail: enzo.nisoli@unimi.it
The mitochondrial lifecycle (mitochondrial biogenesis, dynamics, and removal by mitophagy) is carefully orchestrated to ensure the efficient generation of cellular energy and to maintain reactive oxygen species (ROS) production within an optimal range for cellular health. Based on latest research, these processes largely depend on mitochondrial interactions with other cell organelles, so that the ER- and peroxisome-mitochondrial connections might intervene in the control of cellular energy flow. Damaged organelles are cleared by autophagic mechanisms to assure the quality and proper function of the intracellular organelle pool. Nitric oxide (NO) generated through the endothelial nitric oxide synthase (eNOS) acts a gas signaling mediator to promote mitochondrial biogenesis and bioenergetics, with a favorable impact in diverse chronic diseases of the elderly. Obesity, diabetes and aging share common pathophysiological mechanisms, including mitochondrial impairment and dysfunctional eNOS. Here we review the evidences that eNOS-dependent mitochondrial biogenesis and quality control, and possibly the complex interplay among cellular organelles, may be affected by metabolic diseases and the aging processes, contributing to reduce healthspan and lifespan. Drugs or nutrients able to sustain the eNOS-NO generating system might contribute to maintain organelle homeostasis and represent novel preventive and/or therapeutic approaches to chronic age-related diseases.

Keywords: mitochondrial biogenesis, peroxisomes, endoplasmic reticulum, aging, nitric oxide, endothelial NO synthase (eNOS), reactive oxygen species, hormesis

\section{INTRODUCTION}

Nutrition and medical advancements leading to increased lifespan are not adequately translating into improved healthspani.e., into increased number of healthy, fully functional yearsin modern societies (Valerio et al., 2011). Particularly, the high prevalence of non-communicable diseases and frailty are major concerns in elderly people (Hunter and Reddy, 2013). Globally, the problems are expected to worsen: the World Health Organization estimates that nearly one in four people will be older than 60 by 2050. It is also estimated that approximately one in three adults is obese in Western countries (Ogden et al., 2014). Both aging and obesity are major risk factors for several diseases such as diabetes, heart disease, stroke and certain types of cancers. Gaining wellbeing at all stages of life and maximizing healthy life expectancy are major goals of the post-2015 sustainable agenda (Hunter and Reddy, 2013).

Present-day gerontology research suggests that, unlike traditional approaches that focus on specific diseases, deciphering, and targeting the aging process itself could be the most clever approach toward increased healthspan. Nonetheless, the emerging epidemics of obesity and diabetes (whose prevalence at all ages is growing due to unhealthy habits and economic crisis) can counteract the benefits of reducing chronic disorders throughout the world. Preventing the metabolic consequences of inadequate diet through groundbreaking transdisciplinary investigation should be a primary research focus (Nisoli and Valerio, 2014).
Multiple cell effectors work together to cause the senescent cell phenotype. Particularly, two cellular organelles-nucleus and mitochondrion-have been implicated in the "wear and tear" aspects of aging (Nisoli and Valerio, 2014). Other organelles, including the endoplasmic reticulum (ER), peroxisomes and lysosomes, have been studied in relation to aging mechanisms. Since cellular organelles act in a coordinated manner, we have recently proposed an aging organelle network theory, exploring the possibility that the complex interplay among mitochondria and other cellular organelles may be affected by the aging process (Nisoli and Valerio, 2014). An urgent challenge is to uncover the molecular mechanism(s) regulating the endomembrane systems in cellular aging. Here we review the recent evidence indicating the relevant role played by the nitric oxide (NO) signaling system in the improvement of the age-related metabolic disorders and in healthspan extension. Moreover, we will summarize the current knowledge regarding the role of NO in organelle crosstalk and quality control.

\section{HEALTHY EFFECTS OF CALORIE RESTRICTION AND EXERCISE: THE ROLE OF ENOS AND MITOCHONDRIAL BIOGENESIS NITRIC OXIDE, REACTIVE NITROGEN SPECIES AND THE HORMETIC EFFECT}

NO is highly lipophilic, hyperreactive, diffusible free radical gas (Dudzinski et al., 2006) endowed with a short half-life 
in biological fluids and acting as a fundamental cell signaling molecule in critical physiological processes (Moncada et al., 1991; Pacher et al., 2007). When reacting with superoxide $\left(\mathrm{O}_{2}^{\bullet-}\right)$, NO can give rise to reactive nitrogen species (RNS) which, similarly to reactive oxygen species (ROS) can mediate cellular damage in a wide range of conditions (Pacher et al., 2007) (Figure 1). Traditionally, ROS are represented as unwanted by-products of mitochondrial oxidative phosphorylation and major players in decreased lifespan, based on the Harman's free radical theory of aging (Harman, 2009). With the aim to postpone the aging decay, great efforts have been made to prevent ROS formation by administering high doses of natural or synthetic antioxidant compounds (Ristow and Zarse, 2010). However, several prospective clinical trials designed to demonstrate the health-promoting effects of diverse antioxidants failed, at best showing no benefits (vitamin $\mathrm{C}$ and selenium) or even promoting diseases or increasing mortality in humans (beta carotene, vitamin A, and vitamin E) introducing the so-called "antioxidant paradox" (Ristow and Zarse, 2010; Li et al., 2014; Nisoli and Valerio, 2014). Present evidence does not support antioxidant supplements for primary or secondary disease prevention (Bjelakovic et al., 2012). Indeed, the latest findings (in contrast to Harman's original hypothesis) suggest that-within specific concentrations and exposure-time intervals-ROS behave as key signaling molecules potentially involved in lifespan (Kaelin, 2005; Loh et al., 2009; Ristow et al., 2009; Ristow and Zarse, 2010; Ristow and Schmeisser, 2014), possibly by upregulating endogenous antioxidant levels in the human body. The "mitochondrial hormesis" or "mitohormesis" hypothesis suggest that strategies activating low-level stress may indeed favor longevity (Tapia, 2006; Ristow and Schmeisser, 2014) (Figure 1). Lately, supplying weak pro-oxidants, with the aim to promote the composite endogenous antioxidant response, has been suggested as a more useful approach to the treatment and prevention of diseases (Halliwell, 2013).

\section{PROLONGEVITY MECHANISMS ACTIVATED BY CALORIE RESTRICTION}

In 2003, endogenous NO produced at low doses by endothelial NO synthase (eNOS) was firstly demonstrated to promote mitochondrial biogenesis in several cells and tissues (Nisoli et al., 2003 , 2004). Since then, eNOS and downstream NO signaling are appreciated as major metabolic determinants for the regulation of mitochondrial biogenesis (Scarpulla, 2008). The downstream induction of the expression of peroxisome proliferatoractivated receptor $\gamma$ coactivator $1 \alpha(\mathrm{PGC}-1 \alpha)$ is essential for this effect (Nisoli et al., 2003). It has been established that $\mathrm{NO}$-induced mitochondrial biogenesis requires cyclic guanosine mono-phosphate (cGMP) (Nisoli et al., 2003; Kalogeris et al., 2014). As a consequence of NO-cGMP production, key nuclearencoded mitochondrial biogenesis transcription factors (including nuclear respiratory factor- 1 and mitochondrial transcription factor A) are induced, mitochondrial DNA (mtDNA) levels are increased and mitochondrial function is activated (Nisoli et al., 2003 , 2004). Besides this, NO is able to activate PGC- $1 \alpha$ via AMPK or in a p53-dependent manner, with a possibility of multiple overlapping pathways (Komen and Thorburn, 2014). Further, NO acting via cGMP induces the expression of the SIRT1 deacetylase (Nisoli et al., 2005) a well-known PGC-1 $\alpha$ activity inducer, as discussed below. Clear establishment of the full NO-dependent

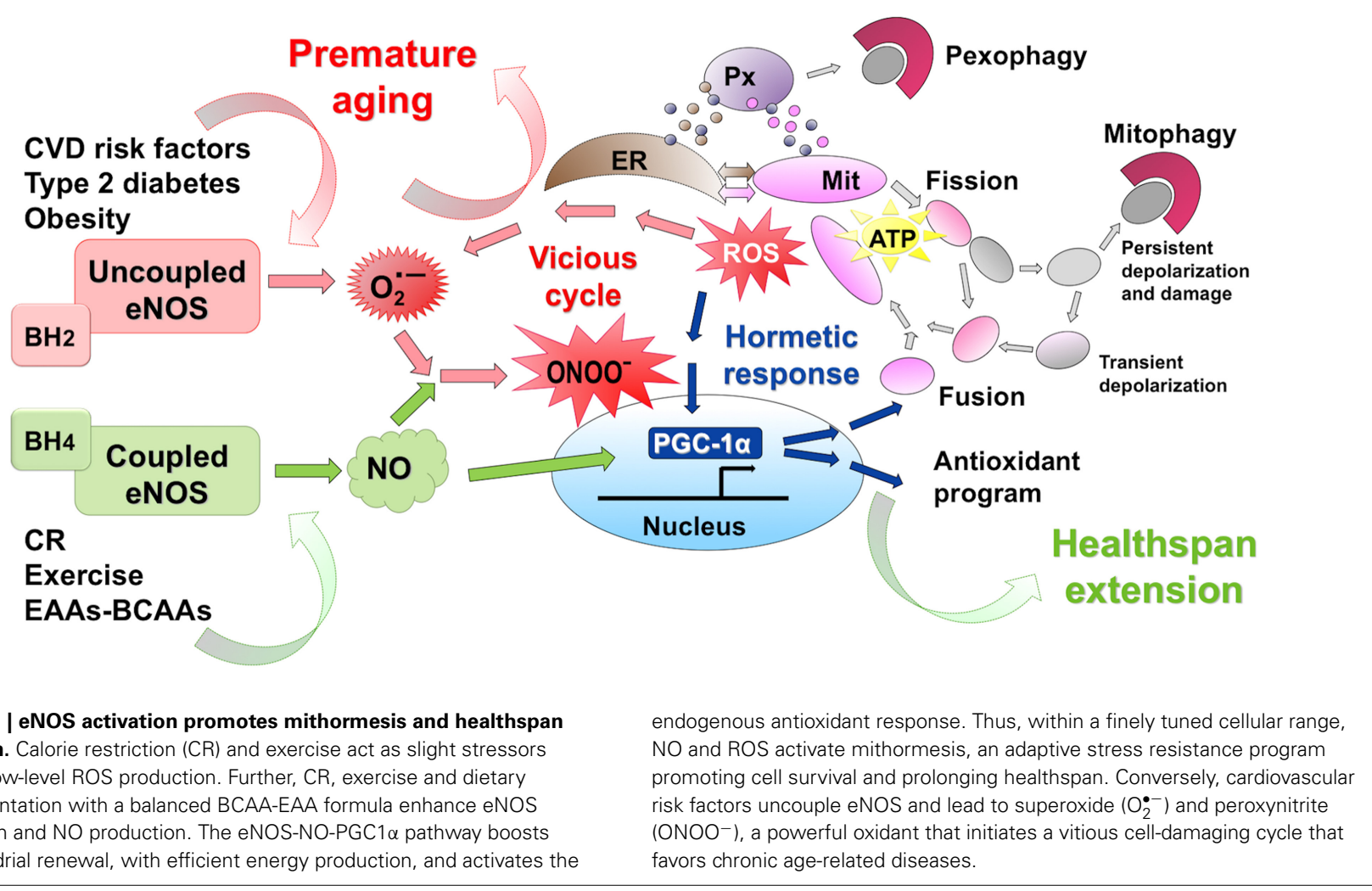


pathway involved in these phenomena is an interesting point awaiting further research.

Subsequently it has been reported that eNOS expression is induced, and NO-dependent mitochondrial biogenesis is augmented, in the every-other-day feeding model of calorie restriction (CR) in mice (Nisoli et al., 2005). Chronic CR without malnutrition, acting as a low-energy stress condition, is considered the gold standard intervention to extend lifespan in most species. Although the possibility that CR could promote lifespan extension in humans is currently debated, the impact of CR in promoting healthspan by attenuating age-related disease is a consistent finding (Fontana et al., 2010). Calorie-restricted mice had upregulated PGC- $1 \alpha$ and sirtuin 1 (SIRT1, the mammalian deacetylase ortholog of the life-extending yeast gene Sir2) and induced endogenous antioxidant response (see also details below) (Nisoli et al., 2005). It should also be mentioned that, although preliminary evidence suggests that high SIRT1 expression is not required for CR-induced lifespan extension (Mercken et al., 2014), its activation extends survival of male mice on a standard diet (Mercken et al., 2013). Interestingly, both SIRT1 and the mitochondrial homologous protein SIRT3, which is also induced by $\mathrm{CR}$, increase eNOS and PGC-1 $\alpha$ biological activity through protein deacetylation (Mattagajasingh et al., 2007; Gouspillou and Hepple, 2013; Guarente, 2013). Additionally, CR induces nuclear localization of SIRT1 in a mechanism requiring eNOS, so that SIRT1 and eNOS may comprise a mutually reinforcing activity loop (Guarente, 2013).

Notably, the CR effects were blunted in eNOS-null $\left(\mathrm{eNOS}^{-/-}\right.$) mice (Nisoli et al., 2005), a strain exhibiting metabolic dysfunctions, age-related diseases and shortened lifespan (Valerio et al., 2011). The mitochondrial biogenic effect of CR has been recently challenged in different rodent models (Hancock et al., 2011; Lanza et al., 2012) particularly due to the lack of demonstration of increased mitochondrial protein synthesis rate in vivo (Miller et al., 2012). However, evidence from diverse groups support the initial findings, demonstrating that CR increases PGC- $1 \alpha$ and the entire paradigmatic transcriptional activation involved in mitochondrial biogenesis, as well as key proteins and/or other relevant markers [including mtDNA abundance and mitochondrial number] in flyes (Zid et al., 2009), rodents (Sreekumar et al., 2002; López-Lluch et al., 2006; Civitarese et al., 2007; Cerqueira et al., 2011) and humans (Civitarese et al., 2007; Mercken et al., 2013). The above reported controversy is likely amplified by virtue of the different CR protocols (percent decrease of ad libitum feeding vs. alternate-day-fasting and/or animal species or strain difference), an issue to be further elucidated to fully understand the complexities herein (Gouspillou and Hepple, 2013).

That NO may extend lifespan has been supported by the relevant observations by $\mathrm{Li}$ and colleagues (Li et al., 2011). By screening several high-NO yeast mutant strains and selecting the top NO producers, the authors showed a positive correlation between increased NO production, mitochondrial respiration, and longevity. Moreover, treatment with the NO donor S-nitrosoglutathione extended yeast lifespan, in part by increasing stress responses, possibly via inducing a hormetic effect. While CR significantly increases NO production in yeast, some highNO yeast mutants only partially mimicked CR, suggesting that activation of different subsets of stress response pathway or additional mechanisms contribute to CR-mediated life extension in these mutants. Noteworthy in this respect are the recent observations in C. elegans, a roundworm that lacks NOS and compensates for its own deficit of NO production by eating its natural food (bacteria). A recent finding showed that bacterial NO produced inside the worm acts to promote longevity and stress resistance via a defined group of genes that function under the dual control of HSF-1 and DAF-16 transcription factors (Gusarov et al., 2013).

\section{HEALTHSPAN-EXTENDING MECHANISMS OF EXERCISE}

Regular exercise has a beneficial impact against various disorders typically accompany aging, including diabetes, sarcopenia, and osteoporosis, and fosters cardiovascular health (Fontana et al., 2010). Notwithstanding, exercise intensity, duration, and frequency needs to be controlled in the elderly since excessive exercise increases mortality at older ages (de Cabo et al., 2014). Contrary to CR, which is able to delay aging processes resulting in increased mean and maximum lifespan, exercise fails to extend maximum lifespan (Holloszy, 1998; Mercken et al., 2012). However, exercise and CR are mechanistically related to some extent, since both induce mitochondrial biogenesis and autophagy (He et al., 2012), two strictly interconnected cellular processes which are coregulated to replace old inefficient mitochondria with new, fuel-efficient ones (Michel et al., 2012). Because these two health-promoting interventions also display distinct molecular signatures (Mercken et al., 2013), the integration of CR regimens with regular exercise might result in synergistic healthy effects, as reported measuring insulin sensitivity and circulating C-reactive protein in nonobese subjects (de Cabo et al., 2014).

Among the physiological adaptations induced by regular exercise, a major event is the upregulation of eNOS expression and the consequent increase of tissue NO production, an event also measured in real-time through skeletal muscle microdialysis in exercising human subjects (Hellsten et al., 2007). This in turn induces cell glucose uptake in skeletal and cardiac muscle to meet increased metabolic requirement (Zhang et al., 2007; Lee-Young et al., 2010; Vettor et al., 2013). Two recent studies provides strong evidence that the eNOS-NO-PGC-1 $\alpha$-dependent mitochondrial biogenesis plays a crucial role in the metabolic activation taking place in heart and white adipose tissue in response to physical activity (Vettor et al., 2013; Trevellin et al., 2014). At this point, it is of interest to describe the NO biochemistry and eNOS biology and to review their relevance in health preservation.

\section{THE DISCOVERY OF NO AS A GASEOUS MESSENGER}

As early as 1787, Antoine Lavoisier discovered nitrogen and named it azote $(\mathrm{N})$, meaning without life, due to its lack of chemical reactivity and inability to support life when provided as the atmosphere. However, nitrogen is presents in amino acids and in the bases that form DNA and RNA, and the metabolism of nitrogenous compounds is central to the metabolic processes of all living organisms. Unable to utilize inorganic nitrogen, animals rely on plant foods to acquire nitrogen, and arginine from seed proteins is their major nitrogen source. Free arginine within the body also derives from endogenous synthesis and turnover 
of body proteins. Arginine is a so-called conditionally essential amino acid for humans, since its synthesis cannot fully meet the needs under certain pathophysiological conditions, including hypercatabolic responses to stress or injury. Apart from its involvement in protein structure and function, arginine has a role as the precursor for the synthesis of NO (Morris, 2006).

Breakthrough studies conducted around the ' 80 s led to the discovery of NO biological actions. Ferid Murad in 1977 discovered the release of NO from nitroglycerine and its action on vascular smooth muscle (Katsuki and Murad, 1977). In 1987, Salvador Moncada and Louis Ignarro independently demonstrated that the endothelium-derived relaxing factor (discovered by Furchgott and Zawadzki, 1980) is NO (Ignarro et al., 1987; Palmer et al., 1987). NO is produced in vary degrees in the cardiovascular, nervous, digestive and immune systems where it modulates a variety of pathophysiological conditions. NO consists of a single oxygen atom bonded to a nitrogen atom through a chemical bond that exhibits partial double bond and partial triple bond character as a result of an unpaired electron (Dudzinski et al., 2006). As a free radical, the NO molecule has unique reactivity that is responsible for its interaction with numerous targets. As a lipophilic gas, NO acts as an unorthodox messenger molecule, which readily diffuses and traverses multiple cell membranes to signal at some distance from its source (Dudzinski et al., 2006).

The effects of NO are mediated by activation of the hemecontaining soluble guanylyl cyclase (sGC), with formation of cGMP (Katsuki and Murad, 1977). Studies involving bacterial proteins from the heme-nitric oxide/oxygen (H-NOX) binding family of gas receptors (sharing high sequence homology and conserved residues with the sGC heme pocket) help understanding the mechanism of sGC activation. Crucial to NO-dependent activation of H-NOX proteins is the formation of a fivecoordinate NO complex (Herzik et al., 2014). High-resolution crystal structures of a bacterial H-NOX protein in the unligated and NO-bound state show that NO binding leads to a pronounced conformational change in the protein as a result of structural rearrangements in the heme pocket (Herzik et al., 2014). NO can also signal independent of cGMP, by (i) competitively inhibiting cytochrome $\mathrm{c}$ oxidase, the terminal heme-containing enzyme in the mitochondrial electron transport chain; (ii) interacting with critical thiols in proteins leading to S-nitrosation, which regulates the function of many proteins; (iii) reacting with superoxide, thus forming RNS and promoting tyrosine nitration of amino acids, which can also affect protein function (Larsen et al., 2012).

\section{ADVANCES TOWARD A BETTER UNDERSTANDING OF ENOS}

NO is produced by three NOS isoforms: the neuronal (nNOS, alternatively designated NOS1 as it was the first NOS isoform to be discovered), inducible (iNOS or NOS2), and endothelial (eNOS or NOS3) ones. Names of nNOS and eNOS derived from the tissues in which they were first identified. NOS isoforms differ in their expression, subcellular localizations and mechanistic features, which are responsible for their unique pathophysiological roles. eNOS is classified as a L-arginine, NADPH: oxygen oxidoreductase, utilizing flavins, and tetrahydrobiopterin (BH4) as cofactors and forming homodimers during activation (Förstermann and Sessa, 2012). The enzymatic reaction generating NO involves the transfer of electrons from NADPH, via the flavins in the Cterminal reductase domain, to the heme in the $\mathrm{N}$-terminal oxidase domain, where the substrate L-arginine is oxidized to L-citrulline and NO (Qian and Fulton, 2013). To efficiently produce NO, eNOS must effectively coordinate the binding of multiple substrates and cofactors. Disruption of this highly coordinated catalysis can result in the production of superoxide and peroxynitrite (Dudzinski et al., 2006).

Constitutively expressed in the endothelium, eNOS is responsible for the formation of vascular NO under physiological condition, and is induced by shear stress or chemical stimuli. The enzyme is activated by increased intracellular $\mathrm{Ca}^{2+}$, and by a complex integration of transcriptional/post-transcriptional mechanisms and post-translational modifications (serine and tyrosine phosphorylation; S-nitrosylation, acetylation) as well as by substrate and cofactor availability and protein-protein interaction. eNOS is enriched in caveolae (the small invaginations of the plasma membrane, especially abundant in endothelial cells and adipocytes) which may serve as microdomains for optimized NOsGC-cGMP signaling (Tsai et al., 2012). Further, native eNOS can shuttle between distinct subcellular domains, having been detected in the cytosol, Golgi apparatus, mitochondria, and even in the nucleus of endothelial cells (Feng et al., 1999), brown adipocytes (Giordano et al., 2002) and other cells, paralleling sGC localization (Gobeil et al., 2006). Comprehensive reviews have been published on eNOS and its regulation and we refer readers to those articles for complete information (Dudzinski et al., 2006; Qian and Fulton, 2013).

Well-characterized actions of NO include the stimulation of vasodilation, vessel formation (Ziche et al., 1994), and mitochondrial biogenesis (Nisoli et al., 2003) as well as inhibition of smooth muscle cell proliferation, leukocyte adhesion, platelet aggregation, and mitochondrial respiration (Qian and Fulton, 2013). Thus, thanks to its antihypertensive, antithrombotic and anti-atherosclerotic properties, eNOS-derived NO represents a key molecule in vasoprotection (Förstermann and Li, 2011). Following the original study demonstrating that eNOS-derived NO promotes mitochondrial biogenesis (Nisoli et al., 2003), the eNOS-NO-PGC-1 $\alpha$ pathway has been shown to be involved in several health-promoting actions. It has been found to be downregulated in adipose tissue of obese mice and rats (Valerio et al., 2006). Consistently, eNOS overexpression exerts anti-obesogenic effects in high fat-fed mice through improved mitochondrial biogenesis and activity in adipose tissue (Sansbury et al., 2012). The eNOS-NO-PGC-1 $\alpha$ pathway is also implicated in anti-aging effects. Besides its activation in calorie-restricted mice (Nisoli et al., 2005) it has an essential role in healthspan extension observed when mice diet is supplemented with a balanced formula of branched-chain amino acids (BCAAs) and other essential amino acids (EAAs) (D'Antona et al., 2010).

Under high oxidative stress in pathological conditions, eNOS may become dysfunctional and contribute to cell damage. In fact, persisting oxidative stress renders eNOS uncoupled (i.e., $\mathrm{O}_{2}$ reduction is uncoupled from $\mathrm{NO}$ synthesis), such that the enzyme no longer produces the vasoprotective NO, but superoxide ( $\mathrm{Li}$ et al., 2014). Among the possible mechanisms involved 
in eNOS uncoupling, depletion of the eNOS cofactor $\mathrm{BH} 4$ (due ROS-dependent oxidation) is likely to play the major role. Another cause of eNOS uncoupling is L-arginine deficiency due to increased arginase activity (Li et al., 2014). Uncoupling of eNOS further creates a vicious cycle (Figure 1), since superoxide and subsequent peroxynitrite overproduction enhances BH4 oxidation and upregulates arginase expression/activity. Preexisting oxidative stress is thus potentiated to the point that it acts as a crucial determinant in cardiovascular disease (Li et al., 2014). This particular issue is becoming an urgent research topic, aimed at identifying therapeutic intervention that optimize eNOS function.

\section{RELEVANCE OF eNOS IN HUMAN DISEASE AND LONGEVITY ENOS DYSFUNCTION AND METABOLIC DISEASES}

Depletion of the eNOS gene induces hyperinsulinemia and insulin resistance (Duplain et al., 2001). A body of evidence also demonstrates that reduced NO bioavailability (as a consequence to multiple-level eNOS dysfunctional regulation) is a key mechanism of endothelial dysfunction. The complex relationship between endothelial dysfunction and insulin resistance in diabetes, obesity and metabolic syndrome has recently been reviewed (Huang, 2009). Mice lacking eNOS fail to respond to cold exposure by thermogenesis, and their brown adipose tissue development and function are defective (Nisoli et al., 2003; Huang, 2009). Abnormalities in energy homeostasis due to reduced eNOS-derived NO increase the risk for cardiometabolic diseases. In fact, $\mathrm{eNOS}^{-/-}$mice are considered a model for the metabolic syndrome (Nisoli et al., 2007) because they combine its main defining features, including hypertension, endothelial dysfunction, insulin resistance and obesity. This phenotype is recapitulated in mice bearing unphosphorylatable eNOS mutations at S1176 (Kashiwagi et al., 2013). Conversely, mice bearing phosphomimetic eNOS mutations at S1176, resulting in increased enzyme activity and NO production, show improved insulin sensitivity and resistance to high fat-induced weight gain (Kashiwagi et al., 2013). Not only eNOS ${ }^{-/-}$mice have defective mitochondrial biogenesis and function (Nisoli et al., 2003), but also they fail to activate mitochondrial biogenesis in response to cold, CR, exercise or dietary interventions (Nisoli et al., 2005; D'Antona et al., 2010; Vettor et al., 2013; Trevellin et al., 2014). The consequences of reduced NO bioavailability have been explored also in humans. Downregulation of multiple cell signals converging in the eNOS-NO system occurs in type 2 diabetes mellitus (T2DM), and contributes to increase the vulnerability of diabetic patients to atherosclerosis, hypertension, and coronary heart diseases (Sharma and Khanna, 2013).

\section{ENOS DYSFUNCTION IN HEART FAILURE}

A deficiency of mitochondrial energetics has been documented both in cardiac aging and in heart failure. Changes in eNOS have been described in experimental and human cardiac failure with somewhat mixed results (Carnicer et al., 2013). Chronic eNOS deletion has been found to induce age-related concentric hypertrophy (Barouch et al., 2002) and to worsen pathological left ventricular remodeling after myocardial infarction in mice (Scherrer-Crosbie et al., 2001). Consistently, cardiomyocyte-restricted eNOS restoration attenuated compensatory hypertrophy and improved left ventricular performance after myocardial infarction (Janssens et al., 2004) and protected against chronic pressure overload (Buys et al., 2007). However, $\mathrm{eNOS}^{-/-}$mice displayed modest hypertrophy and preserved function compared to wild-type mice after chronic transverse aortic constriction (TAC), a severe condition inducing eNOS uncoupling and increased ROS production (Takimoto et al., 2005). Thus, while eNOS is usually protective against maladaptive stress, it can paradoxically contribute to cardiac pathology in uncoupled conditions (Carnicer et al., 2013). Recent evidence also supports a role for the scaffolding protein Cav- 3 in regulating heart NO-sGC-cGMP signaling. A severe depression of NOdependent activation of sGC has been reported in the TAC mouse model, partly due to sGC oxidation owing to its altered interactions with Cav-3 and modified subcellular compartmentation (Tsai et al., 2012). Interestingly, eNOS-NO signaling contributes to the beneficial effects of statins, angiotensin converting enzyme inhibitors and AT1 receptor blockers in experimental heart failure (Shimokawa and Tsutsui, 2010).

\section{ENOS ALLELIC VARIANTS IN HUMAN DISEASES}

Among the several eNOS variants so far described, the eNOS D298 and IVS18 + 27C alleles have been found to be significantly associated to T2DM and metabolic syndrome (Monti et al., 2003). In addition, endothelial dysfunction and an atherogenic profile favoring coronary artery disease (CAD) and restenosis after coronary stent implantation were associated with the same eNOS variant (Galluccio et al., 2008). A recent investigation further demonstrates the significant association between eNOS $-786 \mathrm{C}>$ T polymorphism and CAD (Liu et al., 2014). Interestingly, the $-665 \mathrm{C}>\mathrm{T}$ polymorphism in the eNOS gene predicts increased cardiovascular morbidity and mortality independently of blood pressure and other risk factors in a general population of white Europeans (Olivi et al., 2014). Although to be interpreted with caution, these findings suggest that deficient NO generation as a consequence of eNOS gene variation possibly contributes to the pathogenesis of complex diseases. Further research will be necessary to elucidate the role of these variants in the increased cardiometabolic risk.

\section{eNOS GENE VARIANTS IN LONG-LIVING HUMANS}

Exceptional longevity in humans is a complex trait. A number of genetic variants are associated in centenarians and long-living individuals. A recent genome-wide association study on 410 longlivings and 553 young controls has identified the rs10491334 gene variant of the $\mathrm{Ca}^{2+}$ /calmodulin-dependent protein kinase (CAMKIV) (Malovini et al., 2011). Homozygous carriers of rs10491334 have a significant reduction in CAMKIV expression. In vitro analysis established that CAMKIV activates the survival proteins AKT, SIRT1, and FOXO3A (Malovini et al., 2011). The authors found a reduced frequency of carriers of the minor allele among centenarians (Malovini et al., 2011). Thus, the results point to a detrimental role for the rs10491334 allele in aging process due to reduced survival genes. Interestingly, the prolongevity genes activated by CAMKIV (that is AKT, SIRT1, and FOXO3A) all can promote eNOS activity (Puca et al., 2012; Xia et al., 2013), 
thus suggesting that long-living individuals and centenarians are selected among those with high NO production.

\section{METABOLIC DISEASES AND SENESCENCE SHARE MITOCHONDRIAL IMPAIRMENTS}

A few years ago, we described a marked reduction in eNOS expression, mitochondrial biogenesis and ATP production, together with changes in mitochondrial morphology reminiscent of increased fission dynamics, in fat and skeletal muscles of rodents with genetically- or high fat diet-induced obesity (Valerio et al., 2006). Obesity is increasingly being interpreted as a state of premature aging (Tzanetakou et al., 2012; Nisoli and Valerio, 2014). Notably, NO availability as well as the mitochondrial biogenic capacity are reduced in aged tissues (Nisoli and Valerio, 2014). Application of a novel biomarker of aging has recently revealed a strong correlation between high body mass index and the epigenetic age of liver tissue, with reduced expression of nuclear mitochondrial genes that play a role in oxidative phosphorylation and electron transport (Horvath et al., 2014). Proteomic work lately confirms that obesity parallels key metabolic perturbations observed in the aging process (Tzanetakou et al., 2012; de Castro et al., 2013).

Mitochondrial biology has been extensively investigated in elderly subjects. Aged human skeletal muscles display increased mtDNA mutations. Important evidence that hindering mitochondrial pathology restores health and longevity has been presented by Safdar and colleagues, who studied the progeroid mice which express a mtDNA polymerase- $\gamma$ variant, and exhibit elevated mtDNA point mutations, systemic mitochondrial dysfunction, multisystem pathology, and reduced lifespan (Safdar et al., 2011). Interestingly, endurance exercise training rejuvenated mice mitochondrial turnover and respiratory capacity, and abolished symptoms of accelerated aging and premature mortality (Safdar et al., 2011).

Recent studies revealed a novel molecular circuit linking nuclear DNA damage to mitochondrial dysfunction in advanced age, and resulting in organ decline and disease development. Genetically-induced progressive telomere shortening in mice induced p53 activation, which directly repressed PGC- $1 \alpha$ and PGC-1 $\beta$. The deficitary mitochondrial biogenesis and function resulted in a premature aging phenotype, with heart failure and liver dysfunction (Sahin et al., 2011). Interestingly, subsequent work demonstrated that deletion of the telomere binding protein RAP1 suppresses PGC-1 $\alpha$ leading to obesity and insulin resistance (Duong and Sahin, 2013).

The processes of mitochondrial biogenesis, dynamics, and mitophagy - the so-called mitochondrial lifecycle-represent efficient mechanisms ensuring the health of the cellular pool of mitochondria (see below). Changes in mitochondrial dynamics (increased fission/fragmentation vs. fusion/elongation) progressively reducing bioenergetic efficiency have recently been observed in conditions of excess nutrient intake (Liesa and Shirihai, 2013). Therefore, identification of multiple changes in the mitochondrial lifecycle provide further evidence linking hypernutrition/obesity and senescence through shared organelle dysfunctions, and could explain some of the beneficial effects associated with CR (Liesa and Shirihai, 2013; Nisoli and Valerio, 2014).

Noteworthy, cell-based studies showed that eNOS-derived NO delays age-dependent inhibition of telomerase activity, preventing telomere shortening and counteracting the senescence of endothelial cells (Vasa et al., 2000). Further findings confirmed a hierarchical relationship between eNOS activation and the expression and activity of the catalytic subunit of human telomerase (hTERT) (Vasa et al., 2000; Farsetti et al., 2009). Finally, increased NO bioavailability prevents high glucoseinduced endothelial cell senescence (Hayashi et al., 2006), so that eNOS has recently been proposed as an anti-senescence drug target (Hayashi et al., 2014).

\section{eNOS-DERIVED NO AND MITOCHONDRIA-ENDOPLASMIC RETICULUM CONNECTIONS}

The endoplasmic reticulum (ER) is a highly dynamic organelle governing protein post-translational modification, folding, and oligomerization. Disruption of ER homeostasis lead to accumulation of unfolded/misfolded proteins, a condition commonly referred to as "ER stress." To combat ER stress, cells have evolved a highly conserved adaptive stress response referred to as the unfolded protein response (UPR). Numerous findings have convinced researchers that the ER can undergo ultrastructural and functional changes in aged tissues. ER stress is implicated in age-related disorders, due to the progressive impairment of the UPR and to the deterioration of diverse ER chaperoning systems (Brown and Naidoo, 2012). Molecules able to reduce ER stress, by promoting normal protein folding and/or increasing the unveiling of misfolded proteins, have been proposed to prevent chronic age-related diseases including neurodegenerative diseases, diabetes and atherosclerosis (Brown and Naidoo, 2012).

High-resolution microscopy served to demonstrate that functionally and structurally distinct domains in the ER are sites of membrane contacts with other organelles, where the two membranes are closely apposed without fusing, so that the organelles maintain their distinct identities. Peculiar contacts have been described between the ER and the plasma membrane, mitochondria, Golgi, endosomes and peroxisomes, where a variety of cellular events take place. The functional roles of the stable contact sites between the ER and mitochondria (referred to as mitochondria associated membranes, MAMs) have been reviewed in detail (Rowland and Voeltz, 2012). Among these, $\mathrm{Ca}^{2+}$ released by the inositol 1,4,5-triphosphate receptor in the ER is taken up by mitochondria where it is required for efficient bioenergetic functions (Cárdenas et al., 2010). When this $\mathrm{Ca}^{2+}$ transfer is deficitary, macroautophagy is activated to sustain cell survival (Cárdenas et al., 2010). Interorganelle contacts also control mitochondrial biogenesis and dynamics. Mitochondrial fission is driven by DRP1, a cytoplasmic protein recruited to the ER-mitochondria contact sites. Mitofusin 2, promoting mitochondrial fusion, tethers contacts between mitochondria and the ER (de Brito and Scorrano, 2008). Finally, live confocal microscopy has demonstrated that tethered ER and mitochondria coordinately move along the cytoskeleton (Rowland and Voeltz, 2012).

Alterations in ER-mitochondria juxtaposition can compromise lipid metabolism, protein synthesis and folding, 
mitochondrial function and mitochondrial quality control. The possible changes in ER-mitochondria tethering in senescent tissues and their involvement in disease pathogenesis has been so far investigated in the context of neurodegeneration, demonstrating that improvement of the ER-mitochondria crosstalk sustains neuronal bioenergetics and exerts a neuroprotective function (Calì et al., 2013; Nisoli and Valerio, 2014).

The role of eNOS and NO in mediating the ER-mitochondria relationship is just beginning to be explored. Increased NO synthesis by eNOS has been described to induce the ERmitochondrial chaperone GRP78 and regulate $\mathrm{Ca}^{2+}$ fluxes between the mitochondria, the Golgi and the ER, thus conferring organ protection in experimental liver transplantation (Ben Mosbah et al., 2014). It is likely that in the next few years we will witness to exciting developments in this field.

\section{PEROXISOMAL BIOGENESIS AND CONNECTIONS: A ROLE FOR NO?}

Peroxisomes are remarkably plastic organelles that fulfill important functions including fatty acid oxidation, production of tricarboxylic acid cycle intermediates for mitochondria and control of ROS homeostasis (Schrader et al., 2013). Investigation in recent years has characterized the molecular mechanisms associated with peroxisomal biogenesis. The peroxisome membrane originates from specific ER subdomains or from the growth and division of pre-existing peroxisomes, on the basis of a welldefined dynamic sequence: elongation of the organelle membrane precedes its constriction and final organelle fission (Islinger et al., 2012). Proteins of the PEX11 family act as membrane elongation factors, while dynamin-like proteins dictate fission. Notably, mitochondrial fission proteins such as mitochondrial fission protein 1 (Fis1), mitochondrial fission factor (Mff) and peroxisomal and mitochondrial division factor 1 (PMD1) also localize to peroxisomes. In contrast to mitochondria, however, peroxisomes do not undergo fusion (Islinger et al., 2012). PGC-1 $\alpha$-the master transcriptional co-activator of mitochondrial biogenesis-is also able to increase peroxisome biogenesis (Bagattin et al., 2010). Accordingly, our preliminary experiments show that treatment with the NO donor DETA-NO induces PEX11 $\alpha$ and PEX11 $\beta$ expression in differentiated $\mathrm{C} 2 \mathrm{C} 12$ myocytes, thus possibly promoting peroxisome proliferation (Nisoli et al., unpublished). All in all, peroxisomes and mitochondria share key components of their division machineries, and are rejuvenated by common signals and transcriptional pathways (Nisoli and Valerio, 2014).

Peroxisomes and mitochondria interact each other and communicate with other subcellular compartments (Camões et al., 2009). Studies in yeast suggest that protein import into the peroxisome modulates this interplay (Beach et al., 2012). With replicative and chronological aging, the decline in the efficiency of peroxisomal protein import initiates a pro-aging spiral within this endomembrane system (Titorenko and Terlecky, 2011). Though our understanding of how peroxisomes are incorporated into metabolic pathways is just beginning to emerge, the existence of a peroxisome-mitochondria network suggest possible implications for human diseases. Indeed, both organelles are supposed to contribute to age-related pathological conditions including ischemia-reperfusion injury, cancer, T2DM, and neurodegenerative diseases (Camões et al., 2009), in which NO does play relevant roles.

\section{ORGANELLE QUALITY CONTROL IS AFFECTED BY AGING}

Autophagy is a non-specific cellular degradative pathway conserved in eukaryotes to assure intracellular organelle quality. Mitochondrial homeostasis is maintained by several qualitycontrol mechanisms, including the coordinated events of biogenesis, autophagy and the mitochondrial UPR (UPRmt) (Liesa and Shirihai, 2013; Nisoli and Valerio, 2014).

The selective degradation mechanism for mitochondria is mitophagy. The mitochondrial quality-control system is mostly based on fission events, which produce mitochondria with different bioenergetic states. While efficient mitochondria with high membrane potential are reconnected within the functional mitochondrial network through the fusion process, depolarized mitochondria are destroyed by mitophagy (Liesa and Shirihai, 2013). Defects in mitophagy are thought to contribute to age-related pathology, such as Parkinson's disease (Youle and Narendra, 2011).

The mitochondrial form of the Lon protease (mLon) is essential for metabolizing oxidatively damaged mitochondrial proteins. Its peroxisomal counterpart (pLon) plays a role in eliminating damaged peroxisomal proteins. Expression and activity of the Lon proteases declines with age (Titorenko and Terlecky, 2011). Besides being degraded through non-specific autophagy, peroxisomes undergo a specific autophagic process called pexophagy. A global decrease in lysosomal autophagy and a decline in the processes involved in mitochondrial and peroxisomal quality control have been observed in aged tissues (Beach et al., 2012; Settembre et al., 2013). Dysfunctional quality control results in the accumulation of mitochondria with altered morphology and/or membrane potential, whose reduced bioenergetic efficiency heavily contributes to the cumulative damage associated with aging and metabolic diseases (Nisoli and Valerio, 2014).

Indeed, when autophagic mechanism are unable to meet the demand for clearance of damaged intracellular organelles, cell, and organ pathology emerge. In yeast, worms, flies, and mammals, perturbation of autophagy contributes to accumulation of protein aggregates and dysfunctional mitochondria, and takes part to the pathogenesis of various age-dependent chronic diseases resulting in decreased lifespan (Allison et al., 2014). Interestingly, SIRT1-mediated activation of autophagy is essential for lifespan extension by CR in worms (Allison et al., 2014).

The role of NO and its downstream signals in autophagy induction has long been a controversial issue, with studies describing $\mathrm{NO}$ as a suppressor of starvation-induced autophagy (Sarkar et al., 2011), or indicating NO-based signaling as an inducer of LPS-mediated autophagy (Yuan et al., 2009). While NOS inhibition altered mitochondrial dynamics with decreased fusion and increased fission, this treatment was found to exert minimal effects on autophagy in the vasculature (Miller et al., 2013). A recent study demonstrated the role of $\mathrm{NO}$ as an autophagy inducer via its downstream mediator, 8-nitro-cGMP, in inflammatory conditions (Ito et al., 2013). Interestingly, eNOS-derived NO stabilizes the autophagy-related protein unc51 like kinase (ULK1) and negatively regulated 26S proteasome 
functionality (Xing et al., 2014). The NO-mediated increase of ULK1 has been recently described as a novel pathway leading to SIRT1 protein stabilization, though this seems to occur independently of autophagy in endothelial cells (Xing et al., 2014). Nonetheless, given the important implications of autophagy in various diseases of aging (Allison et al., 2014), the role of NO signaling in different types of autophagy (ULK1-dependent or -independent) deserves further investigation.

\section{CONCLUSIONS AND FUTURE PERSPECTIVES}

Much progress has been made in clarifying the mechanisms regulating mitochondrial lifecycle, mitochondrial-organelle communications and organelle quality system. Future challenge is to deeply investigate the endomembrane system changes in aging and metabolic diseases and the contribution of eNOS-derived NO herein. After more than 30 years since its discovery, we are still finding unsuspected evidence on the pathophysiological roles of NO signaling. In particular, eNOS dysregulation takes part to the pathogenesis of complex-trait metabolic and age-related diseases. Strategies to maintain the eNOS-NO-PGC-1 $\alpha$ pathway within a physiological range might restore the impaired mitochondrial bioenergetics of advanced age, possibly mimicking the beneficial effects of CR and moderate exercise (Figure 1). Interestingly, dietary supplementation with a BCAA-enriched EAA formula has been demonstrated to restore impaired cardiac and skeletal muscle mitochondrial biogenesis in an eNOS-dependent manner in aged mice (D'Antona et al., 2010). The BCAA-EAA formula, which is known to exert a variety of beneficial effects in human subjects, prevented oxidative damage, enhanced physical endurance and prolonged mean mice survival (D'Antona et al., 2010; Valerio et al., 2011).

Though still in its infancy, research on the role of the eNOSNO system in the control of cell organelle connections and quality control might reveal exciting avenues for disease treatment in the coming years. The development of novel therapies aiming to preserve eNOS-NO signaling will benefit from the identification of site-specific interaction with the eNOS structure. Efforts to identify druggable eNOS sites are ongoing (Maron and Michel, 2012). Groundbreaking research is also taking into consideration uncoupled eNOS as a druggable target (Li and Förstermann, 2014). Some drugs in clinical use (including statins and various inhibitors of the renin-angiotensin-aldosterone system) have been shown to prevent eNOS uncoupling under experimental conditions. Since eNOS uncoupling is reversible, it should be of interest to identify conventional drugs or food able to recouple eNOS, switching it back to a well-functioning, NO producing enzyme. The eNOS transcription enhancers AVE9488 and AVE3085 are promising molecules also acting as eNOS recouplers (Li and Förstermann, 2014). Resveratrol can reverse eNOS uncoupling by increasing $\mathrm{BH} 4$ producing enzyme, but has poor bioavailability (Li and Förstermann, 2014). Small molecules acting as specific SIRT1-activating compounds (the so-called STACs) possibly intervening in the SIRT1-eNOS activity loop (Guarente, 2013) also deserve to be investigated in this context. Although our knowledge about the therapeutical usability of the proposed eNOS-targeting molecules in the long-term is limited, further research might provide the market with novel pharmaceuticals or nutraceuticals with fundamental health-promoting effects.

\section{ACKNOWLEDGMENTS}

We are grateful to Giuseppe D'Antona and to all members of our laboratories for valuable discussions. Our work was supported by Ministero dell'Istruzione, dell'Università e della Ricerca, Italy (grant 2009E48P9M_001 to Enzo Nisoli; grants 2009E48P9M_003 and SCN-00442 to Alessandra Valerio).

\section{REFERENCES}

Allison, D. B., Antoine, L. H., Ballinger, S. W., Bamman, M. M., Biga, P., DarleyUsmar, V. M., et al. (2014). Aging and energetics' 'Top 40' future research opportunities 2010-2013. F1000Res. 3, 219. doi: 10.12688/f1000research.5212.1

Bagattin, A., Hugendubler, L., and Mueller, E. (2010). Transcriptional coactivator PGC-1alpha promotes peroxisomal remodeling and biogenesis. Proc. Natl. Acad. Sci. U.S.A. 107, 20376-20381. doi: 10.1073/pnas.1009176107

Barouch, L. A., Harrison, R. W., Skaf, M. W., Rosas, G. O., Cappola, T. P., Kobeissi, Z. A., et al. (2002). Nitric oxide regulates the heart by spatial confinement of nitric oxide synthase isoforms. Nature 416, 337-339. doi: 10.1038/416337a

Beach, A., Burstein, M. T., Richard, V. R., Leonov, A., Levy, S., and Titorenko, V. I. (2012). Integration of peroxisomes into an endomembrane system that governs cellular aging. Front. Physiol. 3:283. doi: 10.3389/fphys.2012.00283

Ben Mosbah, I., Duval, H., Mbatchi, S. F., Ribault, C., Grandadam, S., Pajaud, J., et al. (2014). Intermittent selective clamping improves rat liver regeneration by attenuating oxidative and endoplasmic reticulum stress. Cell Death Dis. 5, e1107. doi: 10.1038/cddis.2014.65

Bjelakovic, G., Nikolova, D., Gluud, L. L., Simonetti, R. G., and Gluud, C. (2012). Antioxidant supplements for prevention of mortality in healthy participants and patients with various diseases. Cochrane Database Syst. Rev. 3:CD007176. doi: 10.1002/14651858.CD007176.pub2

Brown, M. K., and Naidoo, N. (2012). The endoplasmic reticulum stress response in aging and age-related diseases. Front. Physiol. 3:263. doi: 10.3389/fphys.2012.00263

Buys, E. S., Raher, M. J., Blake, S. L., Neilan, T. G., Graveline, A. R., Passeri, J. J., et al. (2007). Cardiomyocyte-restricted restoration of nitric oxide synthase 3 attenuates left ventricular remodeling after chronic pressure overload. Am. J. Physiol. Heart Circ. Physiol. 293, H620-H627. doi: 10.1152/ajpheart.01236.2006

Calì, T., Ottolini, D., Negro, A., and Brini, M. (2013). Enhanced parkin levels favor ER-mitochondria crosstalk and guarantee $\mathrm{Ca}^{(2+)}$ transfer to sustain cell bioenergetics. Biochim. Biophys. Acta 1832, 495-508. doi: 10.1016/j.bbadis.2013.01.004

Camões, F., Bonekamp, N. A., Delille, H. K., and Schrader, M. (2009). Organelle dynamics and dysfunction: a closer link between peroxisomes and mitochondria. J. Inherit. Metab. Dis. 32, 163-180. doi: 10.1007/s10545-008-1018-3

Cárdenas, C., Miller, R. A., Smith, I., Bui, T., Molgó, J., Müller, M., et al. (2010). Essential regulation of cell bioenergetics by constitutive $\mathrm{InsP} 3$ receptor $\mathrm{Ca}^{2+}$ transfer to mitochondria. Cell 142, 270-283. doi: 10.1016/j.cell.2010.06.007

Carnicer, R., Crabtree, M. J., Sivakumaran, V., Casadei, B., and Kass, D. A. (2013). Nitric oxide synthases in heart failure. Antioxid. Redox Signal. 18, 1078-1099. doi: 10.1089/ars.2012.4824

Cerqueira, F. M., Laurindo, F. R., and Kowaltowski, A. J. (2011). Mild mitochondrial uncoupling and calorie restriction increase fasting eNOS, Akt and mitochondrial biogenesis. PLoS ONE 6:e18433. doi: 10.1371/journal.pone.0018433

Civitarese, A. E., Carling, S., Heilbronn, L. K., Hulver, M. H., Ukropcova, B., Deutsch, W. A., et al. (2007). Calorie restriction increases muscle mitochondrial biogenesis in healthy humans. PLoS Med. 4:e76. doi: 10.1371/journal.pmed.0040076

D’Antona, G., Ragni, M., Cardile, A., Tedesco, L., Dossena, M., Bruttini, F., et al. (2010). Branched-chain amino acid supplementation promotes survival and supports cardiac and skeletal muscle mitochondrial biogenesis in middle-aged mice. Cell Metab. 12, 362-372. doi: 10.1016/j.cmet.2010.08.016

de Brito, O. M., and Scorrano, L. (2008). Mitofusin 2 tethers endoplasmic reticulum to mitochondria. Nature 456, 605-610. doi: 10.1038/nature07534

de Cabo, R., Carmona-Gutierrez, D., Bernier, M., Hall, M. N., and Madeo, F. (2014). The search for antiaging interventions: from elixirs to fasting regimens. Cell 157, 1515-1526. doi: 10.1016/j.cell.2014.05.031 
de Castro, N. M., Yaqoob, P., de la Fuente, M., Baeza, I., and Claus, S. P. (2013). Premature impairment of methylation pathway and cardiac metabolic dysfunction in fa/fa obese zucker rats. J. Proteome Res. 12, 1935-1945. doi: $10.1021 / \mathrm{pr} 400025 \mathrm{y}$

Dudzinski, D. M., Igarashi, J., Greif, D., and Michel, T. (2006). The regulation and pharmacology of endothelial nitric oxide synthase. Annu. Rev. Pharmacol. Toxicol. 46, 235-276. doi: 10.1146/annurev.pharmtox.44.101802.121844

Duong, M. T., and Sahin, E. (2013). RAP1: protector of telomeres, defender against obesity. Cell Rep. 3, 1757-1758. doi: 10.1016/j.celrep.2013.06.011

Duplain, H., Burcelin, R., Sartori, C., Cook, S., Egli, M., Lepori, M., et al. (2001). Insulin resistance, hyperlipidemia, and hypertension in mice lacking endothelial nitric oxide synthase. Circulation 104, 342-345. doi: 10.1161/01.CIR.104.3.342

Farsetti, A., Grasselli, A., Bacchetti, S., Gaetano, C., and Capogrossi, M. C. (2009). The telomerase tale in vascular aging: regulation by estrogens and nitric oxide signaling. J Appl Physiol 106, 333-337. doi: 10.1152/japplphysiol.91360.2008

Feng, Y., Venema, V. J., Venema, R. C., Tsai, N., and Caldwell, R. B. (1999). VEGF induces nuclear translocation of Flk-1/KDR, endothelial nitric oxide synthase, and caveolin-1 in vascular endothelial cells. Biochem. Biophys. Res. Commun. 256, 192-197. doi: 10.1006/bbrc.1998.9790

Fontana, L., Partridge, L., and Longo, V. D. (2010). Extending healthy life spanfrom yeast to humans. Science 328, 321-326. doi: 10.1126/science.1172539

Förstermann, U., and Li, H. (2011). Therapeutic effect of enhancing endothelial nitric oxide synthase (eNOS) expression and preventing eNOS uncoupling. $\mathrm{Br}$. J. Pharmacol. 164, 213-223. doi: 10.1111/j.1476-5381.2010.01196.x

Förstermann, U., and Sessa, W. C. (2012). Nitric oxide synthases: regulation and function. Eur. Heart J. 33, 829-837, 837a-837d. doi: 10.1093/eurheartj/ehr304

Furchgott, R. F., and Zawadzki, J. V. (1980). The obligatory role of endothelial cells in the relaxation of arterial smooth muscle by acetylcholine. Nature 288, 373-376.

Galluccio, E., Piatti, P., Citterio, L., Lucotti, P. C., Setola, E., Cassina, L., et al. (2008). Hyperinsulinemia and impaired leptin-adiponectin ratio associate with endothelial nitric oxide synthase polymorphisms in subjects with in-stent restenosis. Am. J. Physiol. Endocrinol. Metab. 294, E978-E986. doi: 10.1152/ajpendo.00003.2008

Giordano, A., Tonello, C., Bulbarelli, A., Cozzi, V., Cinti, S., Carruba, M., et al. (2002). Evidence for a functional nitric oxide synthase system in brown adipocyte nucleus. FEBS Lett. 514, 135-140. doi: 10.1016/S00145793(02)02245-7

Gobeil, F., Zhu, T., Brault, S., Geha, A., Vazquez-Tello, A., Fortier, A., et al. (2006). Nitric oxide signaling via nuclearized endothelial nitric-oxide synthase modulates expression of the immediate early genes iNOS and mPGES-1. J. Biol. Chem. 281, 16058-16067. doi: 10.1074/jbc.M602219200

Gouspillou, G., and Hepple, R. T. (2013). Facts and controversies in our understanding of how caloric restriction impacts the mitochondrion. Exp. Gerontol. 48, 1075-1084. doi: 10.1016/j.exger.2013.03.004

Guarente, L. (2013). Calorie restriction and sirtuins revisited. Genes Dev. 27, 2072-2085. doi: 10.1101/gad.227439.113

Gusarov, I., Gautier, L., Smolentseva, O., Shamovsky, I., Eremina, S., Mironov, A., et al. (2013). Bacterial nitric oxide extends the lifespan of C. elegans. Cell 152, 818-830. doi: 10.1016/j.cell.2012.12.043

Halliwell, B. (2013). The antioxidant paradox: less paradoxical now? Br. J. Clin. Pharmacol. 75, 637-644. doi: 10.1111/j.1365-2125.2012.04272.x

Hancock, C. R., Han, D. H., Higashida, K., Kim, S. H., and Holloszy, J. O. (2011). Does calorie restriction induce mitochondrial biogenesis? A reevaluation. FASEB J. 25, 785-791. doi: 10.1096/fj.10-170415

Harman, D. (2009). Origin and evolution of the free radical theory of aging: a brief personal history, 1954-2009. Biogerontology 10, 773-781. doi: 10.1007/s10522009-9234-2

Hayashi, T., Matsui-Hirai, H., Miyazaki-Akita, A., Fukatsu, A., Funami, J., Ding, Q. F., et al. (2006). Endothelial cellular senescence is inhibited by nitric oxide: implications in atherosclerosis associated with menopause and diabetes. Proc. Natl. Acad. Sci. U.S.A. 103, 17018-17023. doi: 10.1073/pnas.0607873103

Hayashi, T., Yamaguchi, T., Sakakibara, Y., Taguchi, K., Maeda, M., Kuzuya, M., et al. (2014). eNOS-dependent antisenscence effect of a calcium channel blocker in human endothelial cells. PLoS ONE 9:e88391. doi: 10.1371/journal.pone.0088391

He, C., Bassik, M. C., Moresi, V., Sun, K., Wei, Y., Zou, Z., et al. (2012). Exerciseinduced BCL2-regulated autophagy is required for muscle glucose homeostasis. Nature 481, 511-515. doi: 10.1038/nature10758
Hellsten, Y., Nielsen, J. J., Lykkesfeldt, J., Bruhn, M., Silveira, L., Pilegaard, H., et al. (2007). Antioxidant supplementation enhances the exercise-induced increase in mitochondrial uncoupling protein 3 and endothelial nitric oxide synthase mRNA content in human skeletal muscle. Free Radic. Biol. Med. 43, 353-361. doi: 10.1016/j.freeradbiomed.2007.02.029

Herzik, M. A., Jonnalagadda, R., Kuriyan, J., and Marletta, M. A. (2014). Structural insights into the role of iron-histidine bond cleavage in nitric oxide-induced activation of H-NOX gas sensor proteins. Proc. Natl. Acad. Sci. U.S.A. 111, E4156-E4164. doi: 10.1073/pnas.1416936111

Holloszy, J. O. (1998). Longevity of exercising male rats: effect of an antioxidant supplemented diet. Mech. Ageing Dev. 100, 211-219. doi: 10.1016/S00476374(97)00140-1

Horvath, S., Erhart, W., Brosch, M., Ammerpohl, O., von Schönfels, W., Ahrens, M., et al. (2014). Obesity accelerates epigenetic aging of human liver. Proc. Natl. Acad. Sci. U.S.A. 111, 15538-15543. doi: 10.1073/pnas.1412759111

Huang, P. L. (2009). eNOS, metabolic syndrome and cardiovascular disease. Trends Endocrinol. Metab. 20, 295-302. doi: 10.1016/j.tem.2009.03.005

Hunter, D. J., and Reddy, K. S. (2013). Noncommunicable diseases. N. Engl. J. Med. 369, 1336-1343. doi: 10.1056/NEJMra1109345

Ignarro, L. J., Byrns, R. E., Buga, G. M., and Wood, K. S. (1987). Endotheliumderived relaxing factor from pulmonary artery and vein possesses pharmacologic and chemical properties identical to those of nitric oxide radical. Circ. Res. 61, 866-879. doi: 10.1161/01.RES.61.6.866

Islinger, M., Grille, S., Fahimi, H. D., and Schrader, M. (2012). The peroxisome: an update on mysteries. Histochem. Cell Biol. 137, 547-574. doi: 10.1007/s00418012-0941-4

Ito, C., Saito, Y., Nozawa, T., Fujii, S., Sawa, T., Inoue, H., et al. (2013). Endogenous nitrated nucleotide is a key mediator of autophagy and innate defense against bacteria. Mol. Cell 52, 794-804. doi: 10.1016/j.molcel.2013.10.024

Janssens, S., Pokreisz, P., Schoonjans, L., Pellens, M., Vermeersch, P., Tjwa, M., et al. (2004). Cardiomyocyte-specific overexpression of nitric oxide synthase 3 improves left ventricular performance and reduces compensatory hypertrophy after myocardial infarction. Circ. Res. 94, 1256-1262. doi: 10.1161/01.RES.0000126497.38281.23

Kaelin, W. G. (2005). ROS: really involved in oxygen sensing. Cell Metab. 1, 357-358. doi: 10.1016/j.cmet.2005.05.006

Kalogeris, T. J., Baines, C., and Korthuis, R. J. (2014). Adenosine prevents $\mathrm{TNF} \alpha$-induced decrease in endothelial mitochondrial mass via activation of eNOS-PGC-1 $\alpha$ regulatory axis. PLoS ONE 9:e98459. doi: 10.1371/journal.pone.0098459

Kashiwagi, S., Atochin, D. N., Li, Q., Schleicher, M., Pong, T., Sessa, W. C., et al. (2013). eNOS phosphorylation on serine 1176 affects insulin sensitivity and adiposity. Biochem. Biophys. Res. Commun. 431, 284-290. doi: 10.1016/j.bbrc.2012.12.110

Katsuki, S., and Murad, F. (1977). Regulation of adenosine cyclic $3^{\prime}, 5^{\prime}$ monophosphate and guanosine cyclic $3^{\prime}, 5^{\prime}$-monophosphate levels and contractility in bovine tracheal smooth muscle. Mol. Pharmacol. 13, 330-341.

Komen, J. C., and Thorburn, D. R. (2014). Turn up the power - pharmacological activation of mitochondrial biogenesis in mouse models. Br. J. Pharmacol. 171, 1818-1836 doi: 10.1111/bph.12413

Lanza, I. R., Zabielski, P., Klaus, K. A., Morse, D. M., Heppelmann, C. J., Bergen, H. R., et al. (2012). Chronic caloric restriction preserves mitochondrial function in senescence without increasing mitochondrial biogenesis. Cell Metab. 16, 777-788. doi: 10.1016/j.cmet.2012.11.003

Larsen, F. J., Schiffer, T. A., Weitzberg, E., and Lundberg, J. O. (2012). Regulation of mitochondrial function and energetics by reactive nitrogen oxides. Free Radic. Biol. Med. 53, 1919-1928. doi: 10.1016/j.freeradbiomed.2012. 08.580

Lee-Young, R. S., Ayala, J. E., Hunley, C. F., James, F. D., Bracy, D. P., Kang, L., et al. (2010). Endothelial nitric oxide synthase is central to skeletal muscle metabolic regulation and enzymatic signaling during exercise in vivo. Am. J. Physiol. Regul. Integr. Comp. Physiol. 298, R1399-R1408. doi: 10.1152/ajpregu.00004. 2010

Li, B., Skinner, C., Castello, P. R., Kato, M., Easlon, E., Xie, L., et al. (2011). Identification of potential calorie restriction-mimicking yeast mutants with increased mitochondrial respiratory chain and nitric oxide levels. J. Aging Res. 2011:673185. doi: 10.4061/2011/673185

Li, H., and Förstermann, U. (2014). Pharmacological prevention of eNOS uncoupling. Curr. Pharm. Des. 20, 3595-3606. doi: 10.2174/13816128113196660749 
Li, H., Horke, S., and Förstermann, U. (2014). Vascular oxidative stress, nitric oxide and atherosclerosis. Atherosclerosis 237, 208-219. doi: 10.1016/j.atherosclerosis.2014.09.001

Liesa, M., and Shirihai, O. S. (2013). Mitochondrial dynamics in the regulation of nutrient utilization and energy expenditure. Cell Metab. 17, 491-506. doi: 10.1016/j.cmet.2013.03.002

Liu, D., Jiang, Z., Dai, L., Zhang, X., Yan, C., and Han, Y. (2014). Association between the $-786 \mathrm{~T}>\mathrm{C}$ 1polymorphism in the promoter region of endothelial nitric oxide synthase (eNOS) and risk of coronary artery disease: a systematic review and meta-analysis. Gene 545, 175-183. doi: 10.1016/j.gene.2013.09.099

Loh, K., Deng, H., Fukushima, A., Cai, X., Boivin, B., Galic, S., et al. (2009). Reactive oxygen species enhance insulin sensitivity. Cell Metab. 10, 260-272. doi: 10.1016/j.cmet.2009.08.009

López-Lluch, G., Hunt, N., Jones, B., Zhu, M., Jamieson, H., Hilmer, S., et al. (2006). Calorie restriction induces mitochondrial biogenesis and bioenergetic efficiency. Proc. Natl. Acad. Sci. U.S.A. 103, 1768-1773. doi: 10.1073/pnas.0510452103

Malovini, A., Illario, M., Iaccarino, G., Villa, F., Ferrario, A., Roncarati, R., et al. (2011). Association study on long-living individuals from Southern Italy identifies rs10491334 in the CAMKIV gene that regulates survival proteins. Rejuvenation Res. 14, 283-291. doi: 10.1089/rej.2010.1114

Maron, B. A., and Michel, T. (2012). Subcellular localization of oxidants and redox modulation of endothelial nitric oxide synthase. Circ. J. 76, 2497-2512. doi: 10.1253/circj.CJ-12-1207

Mattagajasingh, I., Kim, C. S., Naqvi, A., Yamamori, T., Hoffman, T. A., Jung, S. B., et al. (2007). SIRT1 promotes endothelium-dependent vascular relaxation by activating endothelial nitric oxide synthase. Proc. Natl. Acad. Sci. U.S.A. 104, 14855-14860. doi: 10.1073/pnas.0704329104

Mercken, E. M., Carboneau, B. A., Krzysik-Walker, S. M., and de Cabo, R. (2012). Of mice and men: the benefits of caloric restriction, exercise, and mimetics. Ageing Res. Rev. 11, 390-398. doi: 10.1016/j.arr.2011.11.005

Mercken, E. M., Crosby, S. D., Lamming, D. W., Jebailey, L., Krzysik-Walker, S., Villareal, D., et al. (2013). Calorie restriction in humans inhibits the PI3K/AKT pathway and induces a younger transcription profile. Aging Cell 12, 645-651. doi: 10.1111 /acel.12088

Mercken, E. M., Hu, J., Krzysik-Walker, S., Wei, M., Li, Y., McBurney, M. W., et al. (2014). SIRT1 but not its increased expression is essential for lifespan extension in caloric-restricted mice. Aging Cell 13, 193-196. doi: 10.1111/acel.12151

Michel, S., Wanet, A., De Pauw, A., Rommelaere, G., Arnould, T., and Renard, P. (2012). Crosstalk between mitochondrial (dys)function and mitochondrial abundance. J. Cell. Physiol. 227, 2297-2310. doi: 10.1002/jcp.23021

Miller, B. F., Robinson, M. M., Bruss, M. D., Hellerstein, M., and Hamilton, K. L. (2012). A comprehensive assessment of mitochondrial protein synthesis and cellular proliferation with age and caloric restriction. Aging Cell 11, 150-161. doi: 10.1111/j.1474-9726.2011.00769.x

Miller, M. W., Knaub, L. A., Olivera-Fragoso, L. F., Keller, A. C., Balasubramaniam, V., Watson, P. A., et al. (2013). Nitric oxide regulates vascular adaptive mitochondrial dynamics. Am. J. Physiol. Heart Circ. Physiol. 304, H1624-H1633. doi: 10.1152/ajpheart.00987.2012

Moncada, S., Palmer, R. M., and Higgs, E. A. (1991). Nitric oxide: physiology, pathophysiology, and pharmacology. Pharmacol. Rev. 43, 109-142.

Monti, L. D., Barlassina, C., Citterio, L., Galluccio, E., Berzuini, C., Setola, E., et al. (2003). Endothelial nitric oxide synthase polymorphisms are associated with type 2 diabetes and the insulin resistance syndrome. Diabetes 52, 1270-1275. doi: $10.2337 /$ diabetes.52.5.1270

Morris, S. M. (2006). Arginine: beyond protein. Am. J. Clin. Nutr. 83, 508S-512S.

Nisoli, E., Clementi, E., Carruba, M. O., and Moncada, S. (2007). Defective mitochondrial biogenesis: a hallmark of the high cardiovascular risk in the metabolic syndrome? Circ. Res. 100, 795-806. doi: 10.1161/01.RES.0000259591.97107.6c

Nisoli, E., Clementi, E., Paolucci, C., Cozzi, V., Tonello, C., Sciorati, C., et al. (2003). Mitochondrial biogenesis in mammals: the role of endogenous nitric oxide. Science 299, 896-899. doi: 10.1126/science.1079368

Nisoli, E., Falcone, S., Tonello, C., Cozzi, V., Palomba, L., Fiorani, M., et al. (2004). Mitochondrial biogenesis by NO yields functionally active mitochondria in mammals. Proc. Natl. Acad. Sci. U.S.A. 101, 16507-16512. doi: 10.1073/pnas.0405432101

Nisoli, E., Tonello, C., Cardile, A., Cozzi, V., Bracale, R., Tedesco, L., et al. (2005). Calorie restriction promotes mitochondrial biogenesis by inducing the expression of eNOS. Science 310, 314-317. doi: 10.1126/science.1117728
Nisoli, E., and Valerio, A. (2014). Healthspan and longevity in mammals: a family game for cellular organelles? Curr. Pharm. Des. 20, 5663-5670. doi: $10.2174 / 1381612820666140306093651$

Ogden, C. L., Carroll, M. D., Kit, B. K., and Flegal, K. M. (2014). Prevalence of childhood and adult obesity in the United States, 2011-2012. JAMA 311, 806-814. doi: 10.1001/jama.2014.732

Olivi, L., Gu, Y. M., Salvi, E., Liu, Y. P., Thijs, L., Velayutham, D., et al. (2014). The $-665 \mathrm{C}>\mathrm{T}$ polymorphism in the eNOS gene predicts cardiovascular mortality and morbidity in white Europeans. J. Hum. Hypertens. doi: 10.1038/jhh.2014.66. [Epub ahead of print].

Pacher, P., Beckman, J. S., and Liaudet, L. (2007). Nitric oxide and peroxynitrite in health and disease. Physiol. Rev. 87, 315-424. doi: 10.1152/physrev. 00029.2006

Palmer, R. M., Ferrige, A. G., and Moncada, S. (1987). Nitric oxide release accounts for the biological activity of endothelium-derived relaxing factor. Nature 327, 524-526. doi: 10.1038/327524a0

Puca, A. A., Carrizzo, A., Ferrario, A., Villa, F., and Vecchione, C. (2012). Endothelial nitric oxide synthase, vascular integrity and human exceptional longevity. Immun. Ageing 9:26. doi: 10.1186/1742-4933-9-26

Qian, J., and Fulton, D. (2013). Post-translational regulation of endothelial nitric oxide synthase in vascular endothelium. Front. Physiol. 4:347. doi: 10.3389/fphys.2013.00347

Ristow, M., and Schmeisser, K. (2014). Mitohormesis: promoting health and lifespan by increased levels of Reactive Oxygen Species (ROS). Dose Response 12, 288-341. doi: 10.2203/dose-response.13-035.Ristow

Ristow, M., and Zarse, K. (2010). How increased oxidative stress promotes longevity and metabolic health: the concept of mitochondrial hormesis (mitohormesis). Exp. Gerontol. 45, 410-418. doi: 10.1016/j.exger.2010. 03.014

Ristow, M., Zarse, K., Oberbach, A., Klöting, N., Birringer, M., Kiehntopf, M., et al. (2009). Antioxidants prevent health-promoting effects of physical exercise in humans. Proc. Natl. Acad. Sci. U.S.A. 106, 8665-8670. doi: 10.1073/pnas.0903485106

Rowland, A. A., and Voeltz, G. K. (2012). Endoplasmic reticulum-mitochondria contacts: function of the junction. Nat. Rev. Mol. Cell Biol. 13, 607-625. doi: $10.1038 / \mathrm{nrm} 3440$

Safdar, A., Bourgeois, J. M., Ogborn, D. I., Little, J. P., Hettinga, B. P., Akhtar, M., et al. (2011). Endurance exercise rescues progeroid aging and induces systemic mitochondrial rejuvenation in mtDNA mutator mice. Proc. Natl. Acad. Sci. U.S.A. 108, 4135-4140. doi: 10.1073/pnas.1019581108

Sahin, E., Colla, S., Liesa, M., Moslehi, J., Müller, F. L., Guo, M., et al. (2011) Telomere dysfunction induces metabolic and mitochondrial compromise. Nature 470, 359-365. doi: 10.1038/nature09787

Sansbury, B. E., Cummins, T. D., Tang, Y., Hellmann, J., Holden, C. R., Harbeson, M. A., et al. (2012). Overexpression of endothelial nitric oxide synthase prevents diet-induced obesity and regulates adipocyte phenotype. Circ. Res. 111, 1176-1189. doi: 10.1161/CIRCRESAHA.112.266395

Sarkar, S., Korolchuk, V. I., Renna, M., Imarisio, S., Fleming, A., Williams, A., et al. (2011). Complex inhibitory effects of nitric oxide on autophagy. Mol. Cell 43, 19-32. doi: 10.1016/j.molcel.2011.04.029

Scarpulla, R. (2008). Transcriptional paradigms in mammalian mitochondrial biogenesis and function. Physiol. Rev. 88, 611-638. doi: 10.1152/physrev.00025.2007

Scherrer-Crosbie, M., Ullrich, R., Bloch, K. D., Nakajima, H., Nasseri, B., Aretz, H. T., et al. (2001). Endothelial nitric oxide synthase limits left ventricular remodeling after myocardial infarction in mice. Circulation 104, 1286-1291. doi: 10.1161/hc3601.094298

Schrader, M., Grille, S., Fahimi, H. D., and Islinger, M. (2013). Peroxisome interactions and cross-talk with other subcellular compartments in animal cells. Subcell. Biochem. 69, 1-22. doi: 10.1007/978-94-007-6889-5_1

Settembre, C., Fraldi, A., Medina, D. L., and Ballabio, A. (2013). Signals from the lysosome: a control centre for cellular clearance and energy metabolism. Nat. Rev. Mol. Cell Biol. 14, 283-296. doi: 10.1038/nrm3565

Sharma, A. K., and Khanna, D. (2013). Diabetes mellitus associated cardiovascular signalling alteration: a need for the revisit. Cell. Signal. 25, 1149-1155. doi: 10.1016/j.cellsig.2013.01.022

Shimokawa, H., and Tsutsui, M. (2010). Nitric oxide synthases in the pathogenesis of cardiovascular disease: lessons from genetically modified mice. Pflugers Arch. 459, 959-967. doi: 10.1007/s00424-010-0796-2 
Sreekumar, R., Unnikrishnan, J., Fu, A., Nygren, J., Short, K. R., Schimke, J., et al. (2002). Effects of caloric restriction on mitochondrial function and gene transcripts in rat muscle. Am. J. Physiol. Endocrinol. Metab. 283, E38-E43. doi: 10.1152/ajpendo.00387.2001

Takimoto, E., Champion, H. C., Li, M., Ren, S., Rodriguez, E. R., Tavazzi, B., et al. (2005). Oxidant stress from nitric oxide synthase-3 uncoupling stimulates cardiac pathologic remodeling from chronic pressure load. J. Clin. Invest. 115, 1221-1231. doi: 10.1172/JCI21968

Tapia, P. C. (2006). Sublethal mitochondrial stress with an attendant stoichiometric augmentation of reactive oxygen species may precipitate many of the beneficial alterations in cellular physiology produced by caloric restriction, intermittent fasting, exercise and dietary phytonutrients: "Mitohormesis" for health and vitality. Med. Hypotheses 66, 832-843. doi: 10.1016/j.mehy.2005. 09.009

Titorenko, V. I., and Terlecky, S. R. (2011). Peroxisome metabolism and cellular aging. Traffic 12, 252-259. doi: 10.1111/j.1600-0854.2010.01144.x

Trevellin, E., Scorzeto, M., Olivieri, M., Granzotto, M., Valerio, A., Tedesco, L., et al. (2014). Exercise training induces mitochondrial biogenesis and glucose uptake in subcutaneous adipose tissue through eNOS-dependent mechanisms. Diabetes 63, 2800-2811. doi: 10.2337/db13-1234

Tsai, E. J., Liu, Y., Koitabashi, N., Bedja, D., Danner, T., Jasmin, J. F., et al. (2012). Pressure-overload-induced subcellular relocalization/oxidation of soluble guanylyl cyclase in the heart modulates enzyme stimulation. Circ. Res. 110, 295-303. doi: 10.1161/CIRCRESAHA.111.259242

Tzanetakou, I. P., Katsilambros, N. L., Benetos, A., Mikhailidis, D. P., and Perrea, D. N. (2012). "Is obesity linked to aging?": adipose tissue and the role of telomeres. Ageing Res. Rev. 11, 220-229. doi: 10.1016/j.arr.2011.12.003

Valerio, A., Cardile, A., Cozzi, V., Bracale, R., Tedesco, L., Pisconti, A., et al. (2006). TNF-alpha downregulates eNOS expression and mitochondrial biogenesis in fat and muscle of obese rodents. J. Clin. Invest. 116, 2791-2798. doi: 10.1172/JCI28570.

Valerio, A., D'Antona, G., and Nisoli, E. (2011). Branched-chain amino acids, mitochondrial biogenesis, and healthspan: an evolutionary perspective. Aging 3, 464-478.

Vasa, M., Breitschopf, K., Zeiher, A. M., and Dimmeler, S. (2000). Nitric oxide activates telomerase and delays endothelial cell senescence. Circ. Res. 87, 540-542. doi: 10.1161/01.RES.87.7.540

Vettor, R., Valerio, A., Ragni, M., Trevellin, E., Granzotto, M., Olivieri, M., et al. (2013). Exercise training boosts eNOS-dependent mitochondrial biogenesis in mouse heart: role in adaptation of glucose metabolism. Am. J. Physiol. Endocrinol. Metab. 306, E519-E528 doi: 10.1152/ajpendo. 00617.2013
Xia, N., Strand, S., Schlufter, F., Siuda, D., Reifenberg, G., Kleinert, H., et al. (2013). Role of SIRT1 and FOXO factors in eNOS transcriptional activation by resveratrol. Nitric Oxide 32, 29-35. doi: 10.1016/j.niox.2013.04.001

Xing, J., Liu, H., Yang, H., Chen, R., Chen, Y., and Xu, J. (2014). Upregulation of Unc-51-Like Kinase 1 by nitric oxide stabilizes SIRT1, independent of autophagy. PLoS ONE 9:e116165. doi: 10.1371/journal.pone.0116165

Youle, R. J., and Narendra, D. P. (2011). Mechanisms of mitophagy. Nat. Rev. Mol. Cell Biol. 12, 9-14. doi: 10.1038/nrm3028

Yuan, H., Perry, C. N., Huang, C., Iwai-Kanai, E., Carreira, R. S., Glembotski, C. C., et al. (2009). LPS-induced autophagy is mediated by oxidative signaling in cardiomyocytes and is associated with cytoprotection. Am. J. Physiol. Heart Circ. Physiol. 296, H470-H479. doi: 10.1152/ajpheart.01051.2008

Zhang, Q. J., Li, Q. X., Zhang, H. F., Zhang, K. R., Guo, W. Y., Wang, H. C., et al. (2007). Swim training sensitizes myocardial response to insulin: role of Akt-dependent eNOS activation. Cardiovasc. Res. 75, 369-380. doi: 10.1016/j.cardiores.2007.04.015

Ziche, M., Morbidelli, L., Masini, E., Amerini, S., Granger, H. J., Maggi, C. A., et al. (1994). Nitric oxide mediates angiogenesis in vivo and endothelial cell growth and migration in vitro promoted by substance P. J. Clin. Invest. 94, 2036-2044. doi: 10.1172/JCI117557

Zid, B. M., Rogers, A. N., Katewa, S. D., Vargas, M. A., Kolipinski, M. C., $\mathrm{Lu}$, T. A., et al. (2009). 4E-BP extends lifespan upon dietary restriction by enhancing mitochondrial activity in Drosophila. Cell 139, 149-160. doi: 10.1016/j.cell.2009.07.034

Conflict of Interest Statement: The authors declare that the research was conducted in the absence of any commercial or financial relationships that could be construed as a potential conflict of interest.

Received: 28 November 2014; paper pending published: 23 December 2014; accepted: 12 January 2015; published online: 06 February 2015.

Citation: Valerio A and Nisoli E (2015) Nitric oxide, interorganelle communication, and energy flow: a novel route to slow aging. Front. Cell Dev. Biol. 3:6. doi: 10.3389/ fcell.2015.00006

This article was submitted to Mitochondrial Research, a section of the journal Frontiers in Cell and Developmental Biology.

Copyright (C) 2015 Valerio and Nisoli. This is an open-access article distributed under the terms of the Creative Commons Attribution License (CC BY). The use, distribution or reproduction in other forums is permitted, provided the original author(s) or licensor are credited and that the original publication in this journal is cited, in accordance with accepted academic practice. No use, distribution or reproduction is permitted which does not comply with these terms. 